\title{
Género y pobreza: determinantes de la salud en la vejez
}

\author{
V. Nelly Salgado-de Snyder, D en Bien Soc,(1) Rebeca Wong, D en Econ. ${ }^{(2)}$
}

L a vejez es un constructo social que involucra la asigInación de roles de acuerdo con la edad, por ejemplo, en cuanto a la participación en actividades económicas y sociales, según el género y las normas socioculturales. La vejez debe entenderse como un proceso que involucra una serie de experiencias, muchas de ellas subjetivas, que se encuentran íntimamente relacionadas con las experiencias de vida, la edad biológica y la acumulación de riesgos a lo largo de los años vividos. ${ }^{1,2}$ Las mujeres conforman la mayoría entre los adultos mayores en casi todos los países del mundo. Este fenómeno, conocido como la 'feminización' del envejecimiento, se observa universalmente porque las mujeres llegan a vivir más que los hombres. A pesar de que en el ámbito mundial nacen aproximadamente 105 niños por cada 100 niñas, las mujeres rebasan a los hombres numéricamente alrededor de las edades 30 a 40 años, y dicha ventaja numérica se hace mayor conforme avanza la edad. ${ }^{3} \mathrm{Da}-$ tos de la Organización de las Naciones Unidas (ONU) revelan que globalmente, alrededor del año 2000, había 190 mujeres por cada 100 hombres entre ancianos de mayor edad. Por ejemplo, entre los octogenarios se estiman 181 mujeres por cada 100 hombres, aunque esta proporción aumenta de manera importante para los nonagenarios, con aproximadamente 287 mujeres por cada 100 hombres; y entre los adultos mayores de 100 años en los que se concentran nuevamente las mujeres: 386 mujeres por cada 100 hombres. ${ }^{4,5}$

La Organización Mundial de la Salud (OMS) ${ }^{6}$ ha reportado que, actualmente, más de la mitad de las mujeres en el mundo que tienen 60 años y más viven en países en desarrollo (198 millones comparados con 135 millones de mujeres de los países desarrollados). Las proyecciones demográficas sugieren que el grupo de las mujeres añosas continuará creciendo de forma dramática. En México, la esperanza de vida al nacimiento para la población general es de 75.4 años; 77.9 años para las mujeres y 73.0 años para los hombres. Las diferencias entre hombres y mujeres se acentúan en los estados de la República con menor esperanza de vida, es decir, los más rezagados. ${ }^{7}$

A la fecha, la población de adultos mayores, principalmente los que viven en la pobreza, han recibido muy poca atención por parte de investigadores, prestadores de servicios y diseñadores de políticas públicas. Existe un limitado número de estudios que se han enfocado en la relación entre envejecimiento, género y pobreza. Sobre todo la inequidad persistente a nivel mundial en el acceso a oportunidades para una mejor calidad de vida entre las mujeres ancianas que viven en pobreza.

\section{Problemas más importantes del tema de enfoque}

Las inequidades del envejecimiento y el género están íntimamente vinculadas con la pobreza a través de una interrelación muy compleja. La complejidad de esta relación tridimensional (género, vejez y pobreza) debe ser entendida como el resultado de una secuencia de acciones y experiencias sociales diferenciadas por sexo

(I) Instituto Nacional de Salud Pública, México.

(2) University of Maryland, Estados Unidos. 
que se inicia en edades tempranas y que culminan en la vejez. En el mundo, las mujeres añosas tienden a vivir en pobreza más que los hombres, y el crecimiento de este grupo poblacional con grandes desventajas sociales continúa aumentando de manera importante.

\section{Inequidad de género}

El género debe entenderse como una construcción social no natural que permea todos los rincones del tejido de una sociedad y se refiere al conjunto de características, oportunidades y expectativas que un grupo social asigna a las personas y que éstas asumen como propio, basándose en sus características biológicas, en su sexo. ${ }^{8}$ Los roles de género se construyen desde la infancia a través de la crianza y la educación escolar y familiar, y determinan las actitudes y conductas de hombres y mujeres en la sociedad. De esta forma, el nivel de bienestar de las mujeres y los hombres en la vejez es resultado de la trayectoria de vida que siguieron, así como del contexto social, económico e institucional que los rodeó. De acuerdo con esto, los hombres y las mujeres en la vejez se encuentran en diferentes estados de vulnerabilidad de acuerdo con el rol social y cultural y el nivel de protección institucional que la sociedad les otorga. ${ }^{9}$ Históricamente, la falta de reconocimiento de las relaciones de género como característica fundamental de la jerarquía social ha actuado como una eficiente barrera para abordar adecuadamente los problemas de equidad que enfrentan los hombres y las mujeres, principalmente en la vejez. ${ }^{10}$

Las mujeres en edad de la vejez tienden más a vivir sin una pareja que los hombres. Esto se da como resultado parcial de que, como ya se mencionó anteriormente, en promedio las mujeres vivan más años que los hombres. Además, las mujeres tienden a formar uniones con hombres de mayor edad que ellas, y tienden menos que los hombres a formar nuevas uniones en casos de viudez, separación o divorcio. Por ello, durante la vejez, más mujeres que hombres se encuentran sin pareja, lo que las coloca en una situación vulnerable, tanto desde el punto de vista económico como social. Dicha vulnerabilidad por la falta de una pareja se observa sobre todo en países en desarrollo, donde la participación de las mujeres ancianas en la vida social y económica ha sido sumamente limitada, y su rol social depende, en gran medida, de su situación como hija, esposa o madre a lo largo de su vida.

En México, se da una gran división entre las funciones tradicionales de hombres y mujeres de las cohortes que están actualmente en edad avanzada, y sus consecuencias constituyen la problemática del género en la vejez. Las mujeres se encuentran vulnerables por su relativamente bajo nivel educativo, poca participación en actividades económicas a lo largo de su vida, falta de la pareja durante la vejez y la pérdida económica y de protección institucional que ello puede representar. Para los hombres, la trayectoria de vida en general se resume como de alta participación en actividades económicas, relativamente poca interacción con una red social y familiar, y poca familiaridad con el sistema de salud. Estas dos trayectorias implican una problemática diferente para ambos sexos en la vejez, por lo que las acciones públicas deben dirigirse a hombres y mujeres con énfasis diferentes.

\section{Seguridad social y económica}

Dado que la vejez es una etapa de la vida caracterizada por la disminución de actividades en gran medida laborales, implica también una disminución de los ingresos. En los países en desarrollo sólo una minoría de los ancianos cuenta con los beneficios de un sistema de jubilación. La inseguridad económica es un problema que afecta a todos los ancianos, pero particularmente a aquellos que desarrollaron actividades laborales en el sector informal y que no cotizaron para recibir una jubilación o una pensión en la vejez. Las mujeres ancianas están sobrerrepresentadas en este grupo de adultos mayores que no reciben ingresos. Debido a los roles de género tradicionales, las mujeres tienen menos probabilidad de trabajar para generar ingresos y ahorros que les permitan solventar sus necesidades económicas en la vejez. Por ello, las ayudas familiares se hacen necesarias para apoyar a los adultos mayores.

Recientemente en México se realizó un estudio nacional con dos cohortes de adultos mayores. Los datos de esta encuesta se usan aquí para documentar algunos patrones de comportamiento de esta población. De acuerdo con datos del Estudio Nacional de Salud y Envejecimiento en México 2001 (ENASEM), Wong, Espinoza y Palloni ${ }^{11}$ reportan que $45 \%$ de los hombres de 60 años y más que viven en zonas urbanas cuenta con ingresos por jubilación o pensión, mientras que, en las zonas rurales, sólo 16\% recibe este beneficio. La proporción de mujeres que recibe este tipo de ingresos se reduce considerablemente a $26 \%$ en zonas urbanas y a $10 \%$ en rurales. El mismo estudio revela que las mujeres, más que los hombres, reciben ayuda económica de sus hijos. En las áreas rurales por ejemplo, $72 \%$ de las mujeres de 60 años y más reciben ayuda económica de sus hijos, en comparación con $58 \%$ de los hombres.

\section{Discapacidad y género}

El hecho de que las mujeres tengan vidas más largas no se traduce necesariamente en vidas más sanas, ya que como la probabilidad de desarrollar discapacidades aumenta 
con la edad, la proporción de mujeres con problemas de salud y discapacidad es también mayor. No existen datos que indiquen el nivel de discapacidad de las mujeres en el mundo; sin embargo, estimaciones de la OMS sugieren que las mujeres, tanto de países desarrollados como en desarrollo, pueden esperar vivir más años de vida con discapacidad y limitaciones funcionales que los hombres. ${ }^{6}$ Existen determinantes económicos, sociales, políticos y culturales que influyen en la manera en que las mujeres envejecen con consecuencias inimaginables para su salud y calidad de vida, mismos que se traducen en el incremento de costos a los sistemas de salud. Por otro lado, la situación de los hombres en países en desarrollo es también delicada, ya que las precarias condiciones laborales y las largas y pesadas cargas de trabajo a lo largo de su vida implican un mayor riesgo de discapacidad.

Los datos derivados del ENASEM para México revela que, entre las personas de 60 años y más, una mayor proporción de mujeres que de hombres informa tener limitaciones con actividades de la vida diaria (como comer, bañarse, caminar, usar el excusado, ir a la cama), pero solamente en las áreas urbanas $(8 \%$ para hombres y $14 \%$ para mujeres). En las áreas rurales, la proporción de personas ancianas con limitaciones es similar para hombres y mujeres $(14 \%){ }^{12}$

\section{Problemas de salud}

La trayectoria de vida de los hombres y las mujeres los colocan con una marcada diferencia en la salud en edades de la vejez, que reflejan los estilos de vida que adoptaron a lo largo de su existencia. Debido a su rol social como proveedor económico de la familia, así como a su actividad más intensa en el mercado laboral, los hombres en general pueden estar sujetos a mayores presiones físicas y mentales que ponen en riesgo su organismo. Por ejemplo, los hombres participan más que las mujeres en actividades que conllevan riesgos a la salud como ingerir alcohol, fumar cigarrillos, experimentar con drogas, trabajar horas extras, manejar a gran velocidad, no acudir al médico y no cuidar su dieta, entre otros riegos. Estas situaciones pueden generar en los hombres condiciones cardiovasculares, accidentes laborales y de tránsito, cáncer, enfisema, problemas del hígado, depresión, ansiedad y angustia. Por otro lado, la mujer, por su rol reproductivo, larga carrera de labor doméstica y otro tipo de presiones asociadas con su rol de cuidadora de la unidad familiar, tiene necesariamente un marco epidemiológico muy distinto al del hombre. Así, la carga de la enfermedad en la vejez refleja grandes divergencias por sexo, que van de acuerdo con la acumulación de riesgos asociados a los papeles sociales desempeñados a lo largo de la vida.
El ENASEM indica que, en las áreas urbanas, una mayor proporción de hombres que de mujeres de 60 años y más, reporta haber sido diagnosticado con enfermedad cardiovascular o pulmonar, mientras que una mayor proporción de mujeres reporta alta presión arterial y diabetes. Entre los hombres, $23 \%$ fuma actualmente, comparado con $7 \%$ de las mujeres. ${ }^{11}$

Existen otros factores asociados a la salud y calidad de vida en la vejez entre las mujeres ancianas como la educación y el estado civil. Los niveles de educación y alfabetismo en las mujeres pobres son bajos y se traducen en limitaciones de acceso a los sistemas de protección social. Por otro lado y también asociado a los roles de género y la pobreza es el bajo nivel de escolaridad de las mujeres ancianas en relación con los hombres. Los grupos mas jóvenes se han beneficiado de los programas educativos básicos que desde hace algunas décadas se implementan en nuestro país. En general, el grupo que presenta las condiciones más adversas en cuanto a niveles de alfabetismo es el de las mujeres rurales ancianas. ${ }^{11}$

En relación con el estado civil, las mujeres ancianas en todo el mundo tienden a ser viudas, y la mayoría de adaptan rápidamente a su situación de vida; sin embargo la viudez parece estar acompañada con arreglos de residencia especiales. Las mujeres en la vejez tienden a residir solas, o con hijos, nietos u otros familiares, lo cual puede implicar abusos, violencia y otro tipo de amenazas y riegos para la salud y el bienestar de las mujeres ancianas.

El ENASEM encontró que entre personas de 60 años de edad o más, aproximadamente, $10 \%$ de los hombres y $13 \%$ de la mujeres viven solas, mientras que 35\% de los hombres y $45 \%$ de las mujeres viven en hogares extensos (éstos son conformados por miembros familiares, además de hijos y padres solamente). Las mujeres durante el ciclo de vida realizan el trabajo de cuidadoras del bienestar familiar, puesto que son las que tienden a cuidar primero a los hijos, después a los padres y finalmente al cónyuge enfermo durante la vejez. Sin embargo, la selección del hijo o la hija con la cual residir puede resultar en una situación ventajosa para algunas mujeres en la vejez, pues podría seleccionarse al hijo(a) con mayor capacidad económica para compartir la residencia, lo cual puede representarle a la anciana una mejor calidad de vivienda que lo que podría solventar con su cónyuge o sola. ${ }^{11}$

\section{Servicios de salud}

En muchos países, el acceso a los servicios de atención a la salud está determinado por la participación en el mercado laboral en el sector formal. En los países en desarrollo, las mujeres que han trabajado toda su vida 
para generar ingresos, generalmente, se incorporan a la economía informal y no cotizan en las instituciones que aseguran una vejez libre de problemas o, por lo menos, que otorgan servicios de la salud en la senectud. Para México, los datos del ENASEM muestran que, entre personas de 60 años y más, aproximadamente el mismo porcentaje de hombres y mujeres tienen derechohabiencia (77\% en las áreas urbanas y $40 \%$ en las rurales). Es importante señalar que la población de adultos mayores de 60 años informa tener un mayor nivel de cobertura que los que se encuentran entre los 50 y 59 años, por ejemplo, y esto se debe a que la población de mayor edad tiende a tener cobertura, no solamente por su propia derechohabiencia relacionada con la actividad laboral, sino también por ser padres dependientes de algún asegurado. ${ }^{11}$ De ahí se concluye que la política de cobertura pro familia de las instituciones de seguridad social en México coloca al anciano en situación ventajosa comparada con la de otras generaciones.

Además, tradicionalmente, las mujeres usan los servicios de salud con mayor frecuencia que los hombres a lo largo de su vida, debido a las necesidades de atención derivadas por la reproducción y el cuidado de los hijos. Esta característica puede colocarlas en una posición ventajosa ante los hombres, ya que a edad avanzada, cuando la salud requiere mayor atención, las mujeres pueden estar más familiarizadas con el manejo de su propia enfermedad que los hombres. El ENASEM muestra que entre las personas de 60 años o más, las mujeres refieren que usan servicios preventivos, de hospitalización y visitas al médico con mayor frecuencia que los hombres. ${ }^{12}$

\section{Pobreza}

De acuerdo con el Banco de México, ${ }^{14}$ la distribución del ingreso en el país es una de las más desiguales en el ámbito mundial (13 de 111 países). De acuerdo con datos de la Encuesta Nacional de Ingresos y Gastos de los Hogares realizada en el 2004, aproximadamente 48.6\% de los mexicanos enfrenta pobreza de patrimonio, la cual se refiere a los hogares cuyo ingreso por persona es insuficiente para cubrir el patrón de consumo básico de alimentación, vestido y calzado, vivienda, salud, transporte público, educación y otros bienes. En el interior de este grupo, se encuentra $18.7 \%$ de la población de México con pobreza alimentaria (hogares cuyo ingreso es insuficiente para cubrir las necesidades de alimentación) y el resto enfrenta pobreza de capacidades (hogares cuyo ingreso es insuficiente para cubrir las necesidades de alimentación, educación y salud). La pobreza se manifiesta con mayor intensidad en la población rural de México, en donde se observa una carencia generalizada de recursos económicos, personales, sociales y de oportunidades.

De acuerdo con datos del INEGI, ${ }^{15}$ aproximadamente 25\% de la población de México es rural y se encuentra distribuida en 196000 localidades con menos de 2500 habitantes. Esto nos habla de la gran dispersión poblacional en las regiones rurales del país y de los problemas de costo e infraestructura para llevar servicios de vivienda, educación y salud a este grupo poblacional, lo cual explica parcialmente la concentración de la pobreza en el medio rural. En las regiones pobres de México se observa lo que Frenk y colaboradores ${ }^{16}$ denominan la doble carga epidemiológica, que se refiere a un perfil de enfermedades caracterizadas por ser infectocontagiosas y de la nutrición, pero de forma simultánea aumentan los padecimientos crónico-degenerativos. La situación se agrava porque en zonas rurales no se cuenta con los recursos necesarios para combatirlos. Al llegar a la vejez, las personas pobres enfrentan un estado de salud disminuido como consecuencia de la acumulación de eventos adversos y múltiples carencias del entorno en el que han pasado la mayor parte de su vida.

Recientemente se concluyeron dos investigaciones en México encaminadas a identificar y describir la situación de salud y calidad de vida de hombres y mujeres ancianos que viven en la pobreza rural y urbana en tres estados de la República: Morelos, Guerrero y Jalisco. ${ }^{17-19}$ Entre los hallazgos de ambos estudios resalta que las mujeres en general informaron padecer un mayor número de problemas de salud, lo cual sugiere una mayor multimorbilidad en las mujeres ancianas que viven en pobreza; pero también es un indicador de que las mujeres acuden más al médico y conocen sus padecimientos mejor que los hombres. En ambos estudios, 8 de cada 10 ancianos utilizaban remedios caseros; y 7 de cada 10 usaban medicamentos, sin necesariamente haber sido recetados por un médico. La mayoría de los ancianos consumían una combinación de ambos para tratar sus problemas de salud. Los apoyos sociales y familiares adecuados así como el acceso a los servicios fueron determinantes de la autopercepción de un buen estado de salud. En general, los resultados sugieren que los sistemas informales de apoyo y atención a la salud son los más utilizados por los adultos mayores que viven en pobreza, principalmente, debido a la carencia de recursos personales, económicos y sociales para enfrentar las barreras de un sistema de salud que parece no ser sensible a las necesidades de los grupos más vulnerables. 


\section{Posibles soluciones en el corto, mediano y largo plazos}

Las respuestas a los grandes retos que enfrentamos en torno a la interrelación de la vejez, género y pobreza requieren de un esfuerzo coordinado intersectorial e interdisciplinario en investigación, servicio y políticas públicas. A continuación se enlistan algunas estrategias que podrían ayudar a enfrentar los problemas derivados de esta compleja relación tripartita.

Incentivar la investigación sobre la interrelación de la vejez, el género y la pobreza

Uno de los problemas más importantes en relación con el tema de esta contribución es que, a pesar de que consistentemente los resultados de las encuestas y los proyectos de investigación revelan la persistente inequidad de género y situación de pobreza que impacta de manera negativa la salud de los adultos mayores, no se han llevado a cabo análisis sistemáticos de la interrelación de estos tres factores que sirvan para la elaboración de políticas encaminadas a apoyar a las mujeres ancianas, que son el grupo más vulnerable. Es necesario promover investigaciones estratégicas que respondan a problemas puntuales en torno a la vejez.

\section{Capacitar profesionales de la salud en gerontología}

Actualmente, la proporción de gerontólogos y geriatras en los países en desarrollo es sumamente baja y la respuesta a la demanda es insuficiente. En México hay sólo 250 geriatras en todo el país para atender a 7.3 millones de ancianos. Hay un déficit importante de personal médico especializado para cubrir la demanda de este creciente segmento de la población. Sin embargo, se requieren no únicamente médicos especializados, sino profesionales de otras disciplinas para atender adecuadamente a los ancianos. Es necesario capacitar en gerontología a médicos, psicólogos, oftalmólogos, trabajadores sociales, enfermeras y nutriólogos, entre otros profesionales de la salud. La formación de profesionales en el área de gerontología y geriatría debe tomar en cuenta que las necesidades de atención a la mujer son distintas de las del hombre, especialmente en los ancianos que viven en pobreza crónica.

Asimismo, es necesario destinar una partida presupuestal para la atención de la salud de los adultos mayores, particularmente de aquellos con necesidades especiales. Se ha desentendido de la atención a pacientes geriátricos al no destinarles una partida especial presupuestal.
Desarrollar infraestructura especializada

Existe una escasa infraestructura clínica de los servicios públicos para atender las necesidades de salud de los ancianos que viven en pobreza, como acceso a complementos alimentarios, inmunizaciones y servicios psicológicos. Es necesario responder a las necesidades de salud de los ancianos que viven en pobreza, particularmente en las zonas rurales que no se atienden adecuadamente por falta de apoyos y servicios especializados.

Diseñar políticas públicas en salud, enfocadas a las necesidades de cada sexo

Las trayectorias de vida de los hombres y mujeres adultos mayores en México los colocan en situación vulnerable de manera diferencial, por lo que las políticas públicas deberán diseñarse de manera que se enfoquen a cada sexo por separado. Asimismo, es necesario incluir estrategias a problemáticas comunes como las enfermedades crónico-degenerativas y las lesiones.

Diseñar programas de intervención diferenciales por género

Es necesario desarrollar medidas de prevención de las enfermedades y promoción de la salud con un enfoque diferencial por género. De manera similar, el diseño de programas de prevención de comportamientos de riesgo debe hacerse de forma diferenciada, pues los riesgos que enfrentan hombres y mujeres son diferentes y reflejan los valores socioculturales que contextualizan sus estilos de vida.

Poner en marcha programas de apoyo a las familias de ancianos

El cuidado a los ancianos en México se proporciona en los hogares en forma familiar y la carga de esos cuidados recae sobre todo en las mujeres, quienes cumplen la función de "cuidadoras de otros" que incluye a menores de edad y enfermos. Es necesario que se diseñen programas de apoyo institucional y de salud mental que se aboquen a aligerar la carga asociada al papel de las mujeres ancianas como encargadas del cuidado de otros miembros de la unidad familiar, aún más vulnerables.

\section{Promover el uso de servicios}

Es de fundamental importancia que desde edades más tempranas se fomente el uso de servicios de salud entre hombres y mujeres, principalemente entre aquellos que viven en la pobreza. La utilización temprana de servicios 
se reflejará en un mejor estado de salud durante la vejez y podrá garantizar un mayor y mejor uso de servicios de salud para enfrentar las enfermedades geriátricas. También es importante fomentar el uso de servicios de salud física y mental en lugares de trabajo. La salud ocupacional y la prevención de estilos de vida de riesgo deben fomentarse en los lugares de trabajo.

\section{Dar prioridad a los programas en zonas rurales}

Las diferencias entre los adultos mayores señalan un contraste profundo por área de residencia, lo cual es común para hombres y mujeres. Los programas que enfaticen el bienestar de la población adulta mayor en áreas de marginación rural deben continuar siendo una prioridad nacional.

\section{Diseñar e implementar políticas de prevención}

Las diferencias en la salud de hombres y mujeres ancianas están determinadas en gran parte por su función social y económico y sus estilos de vida. Se espera que éstos vayan cambiando conforme avanza el nivel de desarrollo de los países y conforme las transiciones demográfica y epidemiológica vayan avanzando. Es recomendable que las políticas de salud tengan un énfasis en la prevención de las consecuencias del envejecimiento de las generaciones futuras, que serán distintas a las generaciones actuales. Por ejemplo, en México, las mujeres cada día aumentan su nivel educativo conforme se insertan en mayor número en el mercado laboral. Esta transformación implica además cambios importantes en su estilo de vida y conlleva riesgos a la salud, por lo que deben de adoptarse medidas de prevención, en un primer momento, y posteriormente de corrección. Asimismo, se espera que las generaciones futuras tengan un menor número de hijos, por lo que será necesario tomar en cuenta que los adultos mayores del futuro no tendrán tantas opciones de arreglos residenciales o apoyos familiares, como las alternativas que tienen los ancianos de la generación actual.

\section{Conclusión}

La problemática del adulto mayor en México está caracterizada por la intersección entre las problemáticas de la vejez, el género y la pobreza. Esto se da dentro de un contexto de escasa protección institucional y profundas desigualdades sociales y por áreas urbanas y rurales. En esta contribución se ha planteado que las divergentes trayectorias de vida de los hombres y las mujeres los colocan en riesgos particulares y en situaciones diferentes de vulnerabilidad, por lo que las políticas y programas de salud deben de adoptar una visión de problemas que son comunes a ambos sexos, pero también reconocer que será necesario diseñar políticas separadas por género.

Las posibles soluciones que se han mencionado, sin embargo, no solamente atañen a la población actual de adultos mayores y sus necesidades. Al diseñar programas y políticas para el bienestar de los adultos mayores, debe pensarse en la prevención y la inversión que tiene que hacerse en las generaciones de jóvenes actuales para poder estar en mejor posición de solventar sus necesidades en el futuro. Esto implica el diseño de un grupo de políticas y programas intersectoriales, interdisciplinarios y con visión multigeneracional y a través del tiempo. En esta visión del presente y el futuro, las políticas y programas deben pensarse, sobre todo, en función de que las generaciones de hombres y mujeres adultos mayores del futuro enfrentarán diferentes riesgos que las actuales, y las medidas de prevención pueden anticipar dichos riesgos para lograr dos objetivos: una vejez con mejor calidad de vida para la población y una menor carga social del envejecimiento.

El envejecimiento poblacional es una experiencia que ya han vivido los países desarrollados antes que los países en desarrollo, y este destiempo puede proporcionarles ventajas a los países con menores recursos. Las colaboraciones transnacionales pueden resultar de utilidad para adoptar las lecciones deseables y evitar los errores con consecuencias no deseables para el bienestar de la población de México. El desarrollo de infraestructura, formación de recursos humanos y las actitudes hacia la salud y el envejecimiento multigeneracional con perspectiva de género, son programas de acción que pueden llevar varias décadas, por lo que una planeación inmediata resulta inminente.

\section{Referencias}

I. Bell I.The double standard: age. En: Freeman J, ed.Women: a feminist perspective. California: Mayfield Publishing Co.:237-244.

2. Gist Y,Velkoff V. Gender and aging: demographic dimensions, an international brief by the U.S. Department of Commerce. Bureau of the Census, I-/97-3, 1997,dic:3-8.

3. Kinsella K, Phillips DR. Global aging: the challenge of success. Popul Bull 60(I);2005 mar.

4. United Nations Population Division. World population prospects: the 1998 revision. Nueva York: United Nations, 1999.

5. United Nations Population Fund. The state of the world population 1998. Nueva York: UNFPA, 1998.

6. World Health Organization, Gender, health and aging, 2003 [consultado 2006 sept 14]. Disponible en http://www.who.int/gender/documents/en/ Gender_Ageing.pdf

7. Secretaría de Salud. Salud: México 200I-2005. Información para la rendición de cuentas. México: SSA, 2006. 
8. Browne $C$, ed.Women, feminism, and aging. Nueva York: Springer Publishing Co., 1998:XV-XXIX.

9. Hatch L. Gray clouds and silver linings: women's resources in later life. En: Freeman J, ed.Women: a feminist perspective. California: Mayfield Publishing Co., 1995:180-199.

10. Bury M. Envejecimiento, género y teoría sociológica. En:Arbery S, Ginn J, eds. Relación entre género y envejecimiento. Madrid: Narcea, 1996:35-54. II.Wong R, Espinoza M, Palloni A. Salud de adultos mayores en un contexto socioeconómico amplio: el Estudio Nacional de Salud y Envejecimiento en México. Salud Pública Mex, 2007;49 supl 4:

12. Wong R, Díaz Jj. Health care utilization among older Mexican: health and socioeconomic inequalities. Salud Publica Mex 2007;49 supl 4:

13. Ham-Chande R. El envejecimiento en México: el siguiente reto de la transición demográfica. Tijuana: El Colegio de la Frontera Norte, 2003.

14. Ortiz G. Círculos virtuosos y círculos viciosos: crecimiento y reducción de la pobreza en América Latina. Banco de México, 2006 [consultado 2006 sept I4]. Disponible en: www.banxico.org.mx /gPublicaciones/Seminarios/RelacionesExternas/banxicoFHT.pdf

15. Instituto Nacional de Estadística Geografía e Informática. Estadísticas a propósito del día mundial de la población. 2006 jul II [consultado sept 14]. Disponible en: http://www.inegi.gob.mx/inegi/default.asp?c $=274$
16. Frenk J, Gonález-Block MA, Lozano R. Seis tesis equivocadas sobre las políticas de salud en el combate a la pobreza. Este país [serie en internet], 1998 mar; 84:28-36.

17. Salgado-de SnyderVN. Envejecimiento, género y pobreza en México rural. En: Salgado-de-Snyder VN, Wong R eds. Envejeciendo en la pobreza: género, salud y calidad de vida. Mexico: Instituto Nacional de Salud Pública, 2003:37-56.

18. Salgado-de SnyderVN,Wong RR. Envejecimiento en la pobreza urbana: conclusiones e implicaciones para la salud de los ancianos. En Salgado-de Snyder VN,Wong RR, eds. Envejecimiento, pobreza y salud en población urbana. México: Instituto Nacional de Salud Pública. 2006: I55-16I. 19. Salgado-de SnyderVN, Bojorquez-Chapela I. Estado de salud y utilización de servicios de salud en adultos mayores que viven en pobreza urbana. En Salgado-de Snyder VN,Wong RR, eds. Envejecimiento, pobreza y salud en población urbana. México: Instituto Nacional de Salud Pública. 2006:55-69. 